\title{
The upgrade of the CMS muon system
}

\author{
Marcello Abbrescia* on behalf of the CMS collaboration \\ Universitá and Sezione INFN - via Amendola, 173 - 70126 Bari - ITALY \\ E-mail: marcello.abbrescia@ba.infn.it
}

The CMS muon system is based on three types of gaseous detectors, RPCs, CSCs and DTs. While operating very well in the present conditions, upgrades are foreseen for each of the subsystems, necessary to cope with the increased pile-up, coming along with higher rates and radiation. Electronics can be modernized by profiting from recent developments. In addition, detector R\&D is ongoing, to possibly install other types of detectors, allowing to improve the trigger and tracking capabilities in the forward region. The upgrade programs and the relative R\&D are outlined in this paper

The European Physical Society Conference on High Energy Physics -EPS-HEP2013 18-24 July 2013

Stockholm, Sweden

${ }^{*}$ Speaker. 


\section{Introduction}

The start of the Large Hadron Collider (LHC) operations took place in 2009, and, since then, p-p collisions at a center of mass energy of $7 \mathrm{TeV}$ were collected, increased up to $8 \mathrm{TeV}$ in 2012. During this period, the Compact Muon Solenoid (CMS) experiment [1] has accumulated an integrated luminosity of $29 \mathrm{fb}^{-1}$.

The current planning for the LHC foresees a series of three long shutdowns, designated LS1 (2013-14, currently ongoing), LS2, and LS3. After LS1, the center of mass energy will be increased to be close to $14 \mathrm{TeV}$, with a $25 \mathrm{~ns}$ bunch spacing. Considering that the expected luminosity, immediately after LS1, should be around $1 \times 10^{34} \mathrm{~cm}^{-2} \mathrm{~s}^{-1}$, CMS will experience an average of about 25 inelastic interactions per bunch crossing (referred to as event pile-up, PU), which is the operating scenario for which the experiment was designed.

During the LS2, around 2018, and during the LS3, at the moment foreseen to start in 2022, the injector chain will be improved, and new low $\beta$ and crab cavities will be installed. After LS3, instantaneous luminosity will be leveled to $5 \times 10^{34} \mathrm{~cm}^{-2} \mathrm{~s}^{-1}$ and the PU should exceed 100, for a corresponding integrated luminosity of about $250 \div 300 \mathrm{fb}^{-1}$ per year. This period, expected to last for further ten years at least, is referred to as the High Luminosity phase for LHC (HL-LHC).

CMS was originally designed for ten years of operation at $1 \times 10^{34} \mathrm{~cm}^{-2} \mathrm{~s}^{-1}$, corresponding to a maximum of $500 \mathrm{fb}^{-1}$ of integrated luminosity, while with HL-LHC operation, the experiment will integrate a total luminosity of order $3000 \mathrm{fb}^{-1}$. It is clear that these new conditions, characterized by an integrated luminosity and PU almost an order or magnitude larger than the design values, will be a major challenge for CMS, to be successfully met with detectors that, at the beginning of LS3, will be more than ten years old.

The CMS collaboration is putting a considerable effort to study the issues associated with the HL-LHC operation, and is currently in the phase of defining the optimal strategy to cope with them [2]. For instance, the tracking system and the endcap calorimeters will be upgraded, due to large radiation dose integrated in these regions; also the trigger system will have to be upgraded to assure a constant performance despite the harsher conditions. In this document the plans for the upgrade of the CMS muon system are briefly described.

\section{The present muon system and the motivations for its upgrade}

The present CMS muon system was designed to be robust, efficient and redundant, and to primarily provide muon identification, measurement of the muons transverse momentum, and correct bunch crossing assignment [3]. It makes use of three different chamber technologies, i.e. Drift Tubes (DTs), Cathode Strip Chambers (CSCs), and Resistive Plate Chambers (RPCs) covering the pseudorapidity region $|\eta|<2.4$. In the barrel, divided in five wheels, consisting each of four coaxial stations interleaved with the iron return yoke planes, DT and RPC chambers are installed. The two endcaps, made out of four planar stations each, are instrumented with CSCs and RPCs.

Since the start of the data taking, all three subdetectors have been operating very well, delivering good data for physics and accomplishing all the essential functions for muon triggering and tracking they were designed for [4] [5]. Presently, the muon system performance, and the ones of each subdetector, remains well within the specifications and does not show signs of degradation. 

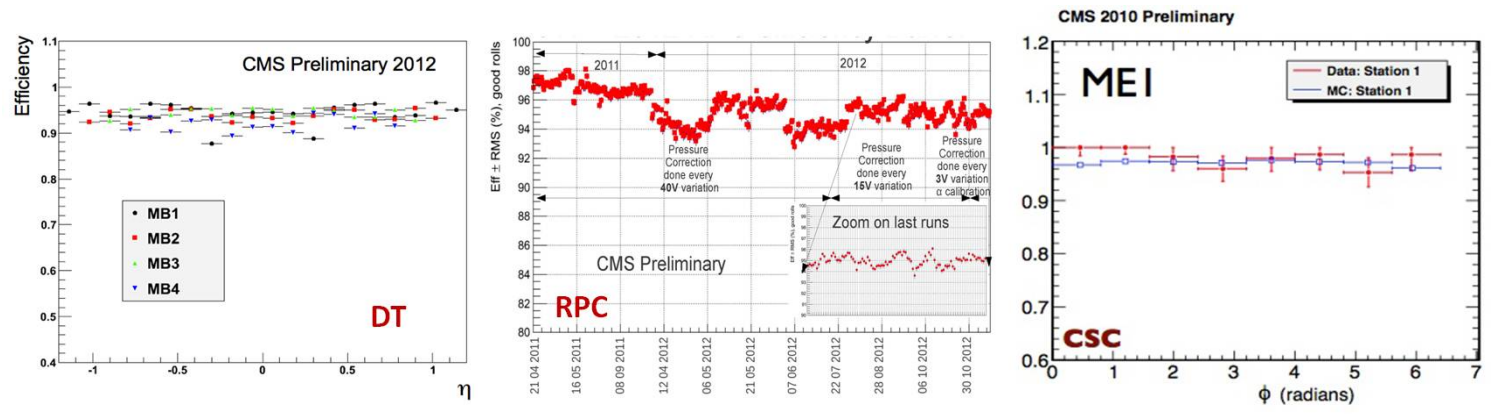

Figure 1: (left) DT average local trigger efficiency in 2012 (lower efficiency points due to the crack between $\mathrm{W}+1$ and W-1); (center) RPC efficiency in 2011-12 (the variations are due to changing of the operating conditions and refining of the calibration algorithm); (right) CSC efficiency for ME1 trigger primitives in 2010 (similar results are obtained for the other stations).

As an example, just three out of the dozens of plots about CMS muon detectors performance are shown in Fig. 1.

Based on the tests performed in the past, it is believed that the installed detectors should survive the whole HL-LHC phase. Detectors in the CMS muon system were tested up to a maximum integrated charge of $0.3 \mathrm{C} / \mathrm{cm}$, a limit that will only be exceeded in some of the most forward CSC chambers at $3000 \mathrm{fb}^{-1}$. Nevertheless, to confirm this performance, a series of tests at the Gamma Irradiation Facility (GIF) at CERN are planned in the near future.

After the end of LS1, muon detection will continue to play a key role in the physics program that will be done with CMS, as muons are one of the most robust signatures of interesting physics processes at the LHC experiments. This will also be true in the most conservative scenario, where no new particle is discovered, and the focus could be just on precision measurements of the Higgs coupling; in fact, muons are critical for measuring both bosonic and fermionic couplings. The importance of the muon system could even increase at the HL-LHC, when the operating conditions for the inner detectors will become more difficult, while at the same time muon systems will suffer the least from non-linear effects of high luminosity.

So, it will be of primary importance to maintain the current performance, in the harsher environment foreseen after LS2 and, more difficult, after LS3. The strategy currently envisioned to maintain the muon system current performance, trigger and offline, despite increasing luminosity and PU, passes from requiring additional redundancy, i.e. installing additional stations, in the most critical regions. The most obvious locations are some existing spaces where RPCs were originally foreseen but not installed. In fact, the present CMS forward muon RPC system consists of three endcap stations and is equipped with chamber up to $|\eta|=1.6$, while the CMS Muon Technical Design Report describes a system with four station and a detector up to to $|\eta|=2.1$ [3].

So, the first natural step to assure the additional redundancy is starting to complete the system, at least up to $|\eta|=1.6$, with the fourth RPC station. This upgrade consists of 144 RPC chambers, to be mounted in two concentric rings (RE4/2 and RE4/3) per station, with 36 chambers per ring [6]. The relative effort of chamber construction has already started and is really world-wide collaborative; bakelite is produced in Italy, gaps are assembled in Korea, chambers mechanics is provided by China, detector electronics is procured in Pakistan, and the final detector assembly is being done in 


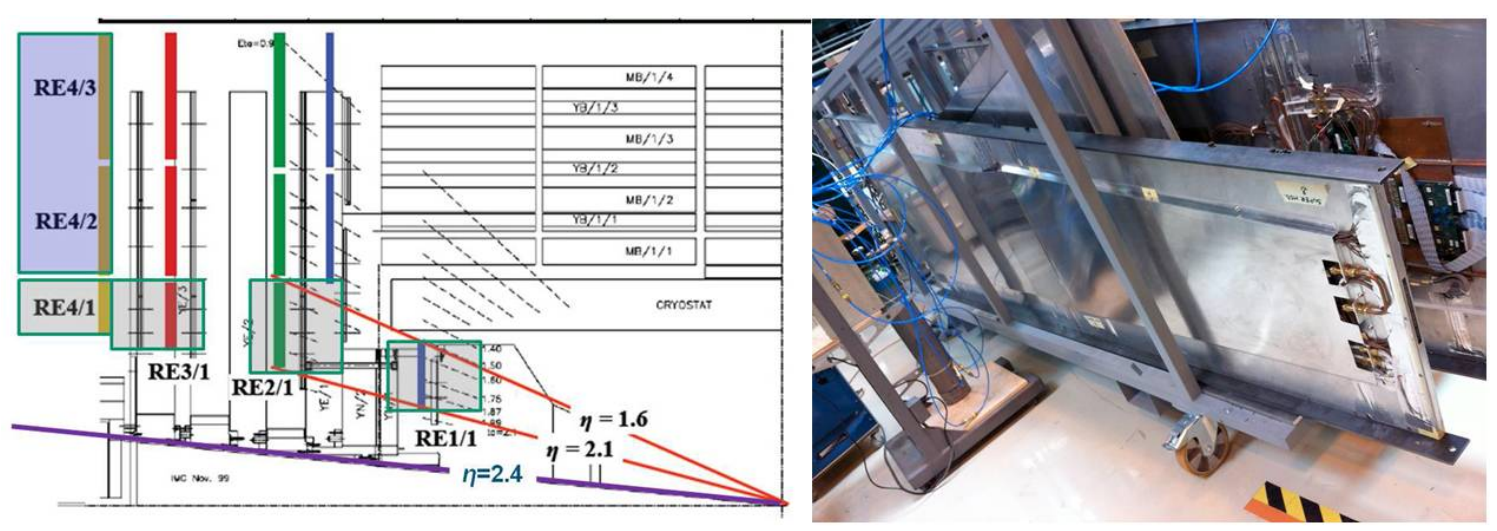

Figure 2: (left) One quarter of the CMS muon system. The colored detectors are the present RPCs. Stations RE4/3 and RE4/2 are being installed during LS1; the remaining stations enclosed in the box are considered for further upgrade during LS2 and LS3. (right) Photograph of the first supermodule (two chambers coupled together) assembled for the RPC LS1 upgrade.

India, Belgium and CERN.

The locations of RE4/2 and RE4/3 are shown in Fig. 2, where also other free spaces potentially exploitable for upgrade are put in evidence. Having RE4/2 and RE4/3 installed will allow the use a "3 (stations) out of 4" trigger logic also in the endcaps, similar to the one already used in the barrel, with a consequential improvement in trigger robustness.

During the LS1, the CSC fourth station will be completed as well, and replacement of some electronics and unganging of the strips of one station will be performed. During the HL phase, the plan is to replace Cathode Front End Boards (CFEBs) if latency will exceed $10 \mu \mathrm{m}$, to avoid any possible data loss.

About the DT system, since from the detector point of view no particular problems are foreseen, most of the work will be about electronics. During the LS1 the installation of a new trigger board is foreseen, as well as a new faster Read Out server. Moreover, the sector collector modules will be moved outside of the cavern to the counting room, replacing copper links with optical ones to cover the increased distance. The goal is to merge the information coming from all subdetectors (DTs, CSCs and RPCs) already in the first level muon trigger, so that also a new Track Finder can be installed. On a longer term, also the DT minicrates will be replaced.

\section{New detectors for the upgrade}

As already pointed out, it could be needed to install other new stations in the most critical parts of the CMS muon system, to keep its performance stable, or, possibly, to improve it. In this case, novel detector technologies could be used. After the completion of the RE4/3 and RE4/2, the available locations are the ones closest to the beam pipe, characterized by a high flux of impinging particles, charged and neutral. Present RPCs, validated up to a rate of about $1 \mathrm{kHz} / \mathrm{cm}^{2}$, could not fulfill the relative requirement on rate capability [7]. In addition, an improved spatial resolution, ranging down to a few hundreds of micrometers, could be very important for triggering, while a time resolution of around 100 ps could be used for background rejection. 


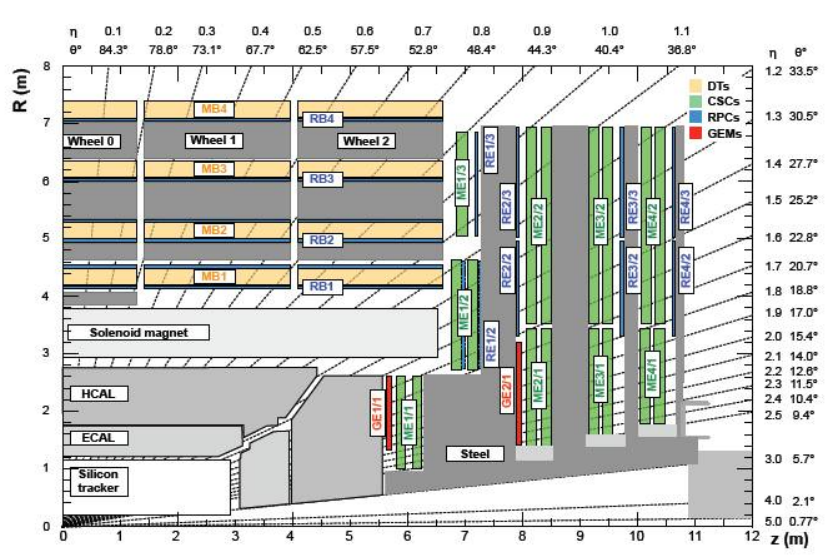

Figure 3: A quadrant of the present CMS muon system, showing DT (yellow), RPC (blue) and CSC (green) chambers. Also the locations of the new GEM stations proposed for the muon system upgrade are indicated in red.

Several projects on new types of high-rate muon detectors have been ongoing in the past years. In particular, an intense R\&D carried out in the framework of the GEMs for CMS (CMS GEM collaboration), started five years ago, has led to the proposal of equipping with triple Gas Electron Multipliers (GEMs) based detectors the two inner stations, conventionally called GE1/1 and GE2/1 in the endcaps of the CMS experiment. They are indicated in Fig. 3.

GEMs are gaseous detectors, characterized by a spatial resolution of order $100 \mu \mathrm{m}$, time resolution of a few ns, and detection efficiency more than $90 \%$ even for rate exceeding few $\mathrm{MHz} / \mathrm{cm}^{2}$ [8]. Despite being invented just in 1997, a long term experience on the field has been accumulated in LHCb, COMPASS and TOTEM; in addition they can be operated with a non-flammable gas mixture $\left(\mathrm{Ar} / \mathrm{CO}_{2} / \mathrm{CF}_{4}\right)$, which is an added value in the underground caverns of the LHC experiments, and GEM foils are currently produced in large areas.

Since the start of this project, many tests have been performed to assess the performance of these detectors. At first, small $10 \times 10 \mathrm{~cm}^{2}$ detectors were used to investigate the achievable time resolution, which resulted to be around $5 \mathrm{~ns}$ (see Fig. 4, left). Later on in 2010, full size prototypes were built, and the relative position resolution, ranging around $150 \mu \mathrm{m}$ was studied (see Fig. 4, right) [9] [10]. Now, the fifth detector generation is being built.

Two techniques were developed or optimized, which are particularly important from the point of view of the future mass production. GEMs foils for CMS are being produced using the singlemask photolitographic hole etching procedure, which overcomes all the issues characteristics of the usual double-mask etching technique and related to the two masks alignment [11]. Moreover, a major improvement in the detector assembly procedure was introduced in 2011 with the socalled stretching technique, in which the GEM foils are mechanically stretched and the detector is constructed without the need of spacers or gluing, which makes it possible to open it again in case repairs are needed [12].

In the present proposed schedule, GE1/1 would be instrumented during LS2, while GE2/1 would be installed during LS3; few chambers are foreseen to be installed, for testing purposes, at the end of 2016. Also the possibility to install GEM-based detectors in an additional station at high 

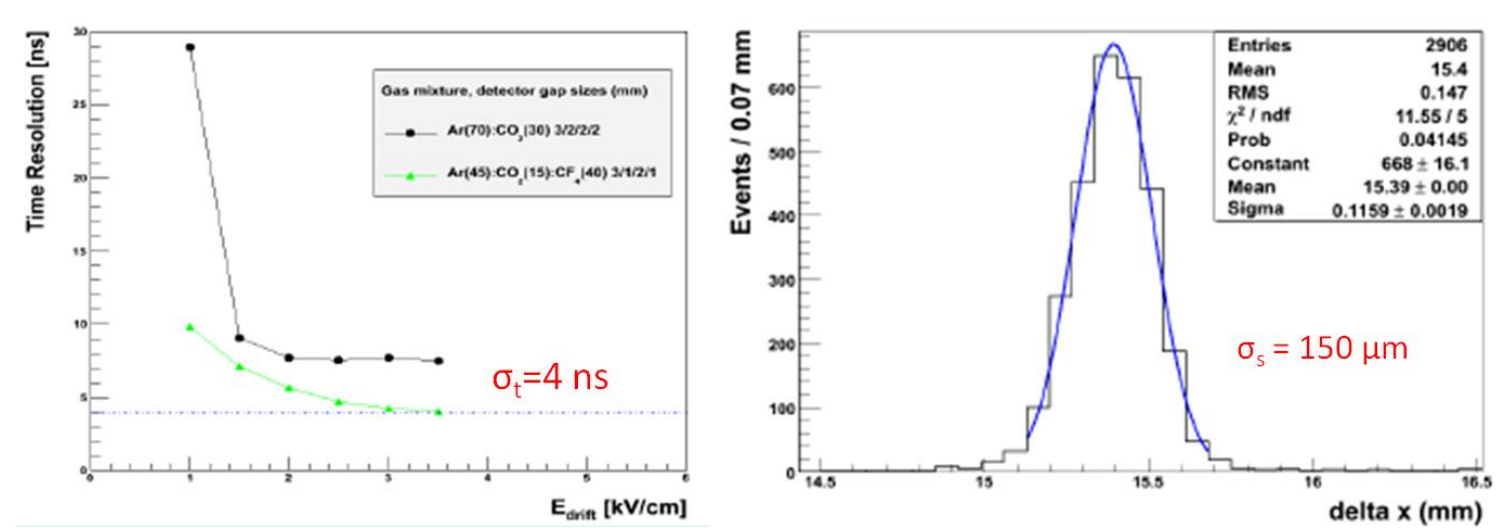

Figure 4: (left) Time resolution with a small size triple GEM detector, measured in 2010; (right) position resolution, measured with the first full GE1/1 full size prototype.

$\eta$ behind the calorimeters is being investigated.

For the remaining two stations (indicated in Fig. 2 as RE3/1 and RE4/1), other solutions are studied. A possibility foresees the use of RPCs with improved rate capability; this can be achieved using different methods, i.e. by lowering the electrode resistivity, or by changing the operating conditions and as a consequence transferring part of the needed amplification from gas to front-end electronics, or by changing the detector configuration, in particular in terms of gap thickness or number.

One project proposed makes use of RPCs made out of glass characterized by a resistivity, around $10^{10} \Omega \mathrm{cm}$, considerably lower with respect to standard float glass [13]. The surface smoothness allows also to use an electronic threshold lower than the one presently used for bakelite RPCs, and, finally, a configuration with three $1.2 \mathrm{~mm}$ thick gaps on each side of the readout strips is proposed, to assure maximum rate capability. The results obtained in various beam tests seem to be very promising, showing a rate capability of a few $\mathrm{kHz} / \mathrm{cm}^{2}$ and a time resolution better than 100 ps.

Also multi-gap RPCs built using standard CMS bakelite are under study, and the relative results are very encouraging [14], and the possibility to use double gap CMS RPCs with a more sophisticated electronics and better shielding is being investigated as well.

\section{Conclusions}

The CMS muon system has been operating extremely well, delivering very good data for physics: after 3 years of LHC at increasing luminosity and 6 years from the end of construction, its performance is within specifications both for triggering and as a reconstruction system.

The conditions after LS2 and, furthermore after LS3, in terms of instantaneous and integrated luminosity, will pose a significant challenge for the system. Therefore, strategies to cope with these new conditions are being developed, so that its performance remains stable despite increased PU, luminosity and background.

Present expectations are that most of the existing detectors should survive for the whole HLLHC period; nevertheless trigger and readout electronics will have to be upgraded, and new de- 
tectors should be installed in the most critical regions, to ensure performance stability or possibly improve it, particularly in terms of acceptance.

To perform a thorough study of possible aging effects of the existing detectors, and to validate the novel ones in conditions as close as possible to the real conditions, adequate testing facilities, like GIF++ currently being instrumented at CERN, are mandatory.

The novel detectors proposed (GEMs or some kind of improved RPCs), provide a full range of characteristics that fit nicely with the ones required for the upgraded CMS muon system experiments, even if, in some cases, some R\&D is still ongoing. Therefore, in principle, where the decision has not been done still, various scenarios, involving different detectors, can be pursued.

A clear overall strategy is essential for optimizing the tight resources, in terms of people and money, which will be available in future for the upgrade of the muon system, while ensuring a smooth operation and a safe data taking to the CMS experiment.

\section{References}

[1] CMS Collaboration, The CMS Experiment at the CERN LHC, JINST 3 (2008) S08004.

[2] CMS Collaboration, Technical Proposal for the Upgrade of the CMS Detector through 2020, CERN-LHCC-2011-006; CMS-UG-TP-1; LHCC-P-004.

[3] CMS Collaboration, The Muon Project, CMS Technical Design Report, CERN/LHCC 97-32; CMS-TDR-003.

[4] CMS Collaboration, The performance of the CMS muon detector in protonproton collisions at $\sqrt{s}=7$ $\mathrm{TeV}$ at the LHC, submitted to JINST.

[5] M. Abbrescia on behalf of the CMS Collaboration, Operation, performance and upgrade of the CMS Resistive Plate Chamber system at LHC, in print on Nucl. Instr. and Meth. A (2013).

[6] M. Tytgat et al., The Upgrade of the CMS RPC System during the First LHC Long Shutdown 2013, JINST 8 T02002

[7] M. Abbrescia, The dynamic behaviour of Resistive Plate Chambers, Nucl. Instr. and Meth. A533 (2004) $7-10$

[8] F. Sauli, Nucl. Instr. and Meth. A386 (1997) 531.

[9] D. Abbaneo et al., Characterization of GEM Detectors for Application in the CMS Muon Detection System, 2010 IEEE Nucl. Sci. Symp. Conf. Rec. 1416-1422; RD51 Note 2010-005

[10] M. Tytgat et al., Construction and Performance of Large-Area triple-GEM Prototypes for Future Upgrades of the CMS Forward Muon System, 2011 IEEE Nucl. Sci. Symp. Conf. Rec. 1019-1025

[11] M. Villa et al., Progress on large area GEMs, Nucl. Instrum. Meth. A 628 (2011) 182

[12] M. Tytgat et al., Construction and Performance of Large-Area triple-GEM Prototypes for Future Upgrades of the CMS Forward Muon System, 2011 IEEE Nucl. Sci. Symp. Conf. Rec. 1019-1025

[13] Y.Wang et al., RPC2010 proceedings, Nucl. Instr. Meth. A661, S134 (2012)

[14] K. S. Lee et al., Tests of multigap RPCs for high- $\eta$ triggers in CMS, 2012 JINST 7 P10009 